\title{
Assessing the surgical outcome of the "chopsticks" technique in endoscopic transsphenoidal adenoma surgery
}

\author{
Carlo Serra, MD, ${ }^{1}$ Victor E. Staartjes, BMed, ${ }^{1}$ Nicolai Maldaner, MD, ${ }^{1}$ David Holzmann, MD, ${ }^{2}$ \\ Michael B. Soyka, MD, ${ }^{2}$ Marco Gilone, MD, ${ }^{3}$ Christoph Schmid, MD, ${ }^{4}$ Oliver Tschopp, MD, ${ }^{3}$ and \\ Luca Regli, MD'
}

1Department of Neurosurgery, Clinical Neuroscience Center; ${ }^{2}$ Department of Otorhinolaryngology, Head and Neck Surgery; and ${ }^{4}$ Department of Endocrinology, Diabetes, and Clinical Nutrition, University Hospital of Zürich, University of Zürich, Switzerland; and ${ }^{3}$ Department of Neurosciences, Reproductive and Odontostomatological Sciences, Division of Neurosurgery, Università degli Studi di Napoli "Federico II," Naples, Italy

OBJECTIVE The "chopsticks" technique is a 3-instrument, 2-hand mononostril technique that has been recently introduced in endoscopic neurosurgery. It allows a dynamic surgical view controlled by one surgeon only while keeping bimanual dissection. Being a mononostril approach, it requires manipulation of the mucosa of one nasal cavity only. The rationale of the technique is to reduce nasal morbidity without compromising surgical results and complication rates. There are, however, no data available on its results in endoscopic surgery (transsphenoidal surgery [TSS]) for pituitary adenoma.

METHODS The authors performed a cohort analysis of prospectively collected data on 144 patients (156 operations) undergoing TSS using the chopsticks technique with 3T intraoperative MRI. All patients had at least 3 months of postoperative neurosurgical, endocrinological, and rhinological follow-up (Sino-Nasal Outcome Test-20 [SNOT-20] and Sniffin' Sticks). The surgical technique is described, and the achieved gross-total resection (GTR) and extent of resection (EOR) together with patients' clinical outcomes and complications are descriptively reported.

RESULTS On 3-month postoperative MRI, GTR was achieved in $71.2 \%$ of patients with a mean EOR of $96.7 \%$. GTR was the surgical goal in 122 of 156 cases and was achieved in 106 of 122 (86.9\%), with a mean EOR of $98.7 \%$ (median $100 \%$, range $49 \%-100 \%$ ). There was no surgical mortality. At a median follow-up of 15 months (range 3-70 months), there was 1 permanent neurological deficit. As of the last available follow-up, $11.5 \%$ of patients had a new pituitary single-axis deficit, whereas $26.3 \%$ had improvement in endocrinological function. Three patients had new postoperative hyposmia. One patient had severe impairment of sinonasal function (SNOT-20 score $>40$ ). The operation resulted in endocrine remission in $81.1 \%$ of patients with secreting adenomas.

CONCLUSIONS This study shows that the chopsticks technique confers resection and morbidity results that compare favorably with literature reports of TSS. This technique permits a single surgeon to perform effective endoscopic bimanual dissection through a single nostril, reducing manipulation of healthy tissue and thereby possibly minimizing surgical morbidity.

https://thejns.org/doi/abs/10.3171/2020.3.FOCUS2065

KEYWORDS pituitary surgery; sinonasal morbidity; chopsticks technique; SNOT-20

$\mathrm{T}$ HE transsphenoidal route is currently established as the preferred surgical approach for pituitary adenomas (PAs), with transcranial surgery being reserved for highly selected cases. ${ }^{3,27}$ Technical nuances of the transsphenoidal approach are regularly being discussed; the use of the endoscope versus the microscope is probably the most frequently debated. ${ }^{24}$

Although class I evidence of the superiority of endoscopes is lacking, endoscopes are increasingly used within the neurosurgical community. ${ }^{28}$ The improved panoramic visualization of anatomical structures is the main argument in favor of this technique. There is, however, no consensus on the technical details of the endoscopic procedure, and several variations have been developed and reported.

In the classic endoscopic technique, originally popularized for sinus surgery by Messerklinger, ${ }^{22}$ the operating surgeon held the endoscope in one hand and a working in-

ABBREVIATIONS EOR = extent of resection; GTR = gross-total resection; iMRI = intraoperative MRI; PA = pituitary adenoma; SNOT-20 = Sino-Nasal Outcome Test-20; STR = subtotal resection; tGTR = GTR as target; TSS = transsphenoidal surgery; tSTR = STR as target.

SUBMITTED January 29, 2020. ACCEPTED March 3, 2020

INCLUDE WHEN CITING DOI: 10.3171/2020.3.FOCUS2065. 
strument in the other. The obvious advantage of bimanual dissection quickly pushed the evolution of the technique either into a 2-surgeon, 3-hand variation or toward the introduction of a mechanical holder for the endoscope. . $^{5,13,20}$ However, the movements of 2 surgeons operating at the same time in such a narrow space must be perfectly coordinated to avoid conflicts between instruments. A mechanical holder does not allow the surgeon to continuously change the position of the endoscope to optimize the surgical view as needed. Moreover, it can significantly obstruct the view if used through a purely mononostril approach. The development of binostril approaches was then the logical consequence, ${ }^{6}$ since they were deemed to increase not only the comfort of the operating surgeon but also the maneuverability of instruments and the surgical field of view. ${ }^{9,10}$ The so-called "two nostrils four hands technique" 7 was then developed with the aim of speeding up the surgical procedure. If, on one hand, a 2-nostril technique allows a wider surgical exposure, on the other, it entails a disruption of healthy mucosa of both nostrils, thereby increasing the surgical footprint of the entire procedure.

The so-called "chopsticks" technique was introduced into endoscopic surgery by Manickavasagam et al. ${ }^{19}$ in 2010 and into the neurosurgical practice by Froelich et al. ${ }^{15}$ The chopsticks technique is a 3-instrument, 2-hand mononostril technique where the operating surgeon holds the endoscope with the nondominant hand together with the suction and the working instrument with the dominant hand (Fig. 1). This approach allows for a dynamic surgical view controlled by only one surgeon without completely losing the advantages and safety of a bimanual dissection (Video 1).

VIDEO 1. Demonstration of the chopsticks technique in endoscopic transsphenoidal pituitary surgery. Copyright Carlo Serra. Published with permission. Click here to view.

Moreover, this technique can be performed through a purely mononostril approach, thereby eliminating manipulation of the second nasal cavity and thus preserving healthy tissues. The rationale of the technique is thus to reduce nasal morbidity without compromising surgical results or increasing the complication rate. However, to date, no data have been available to support this theory.

The goal of the present study was thus to report for the first time in literature the surgical outcome of the chopsticks technique and offer a benchmark for future studies on this topic by prospectively analyzing collected data of a homogeneous series of patients operated on with the chopsticks technique for resection of PAs.

\section{Methods}

\section{Patient Population}

Relevant clinical and radiological data of patients undergoing surgery for a PA in the neurosurgical department of the University Hospital of Zurich have been prospectively collected in a database since October 2012. All patients are treated according to the same PA protocol, which includes the following: complete endocrinological assessment pre- and postoperatively (at discharge, 6 and 12 weeks after the operation, and as needed thereafter), thorough pre- and postoperative neurological examination

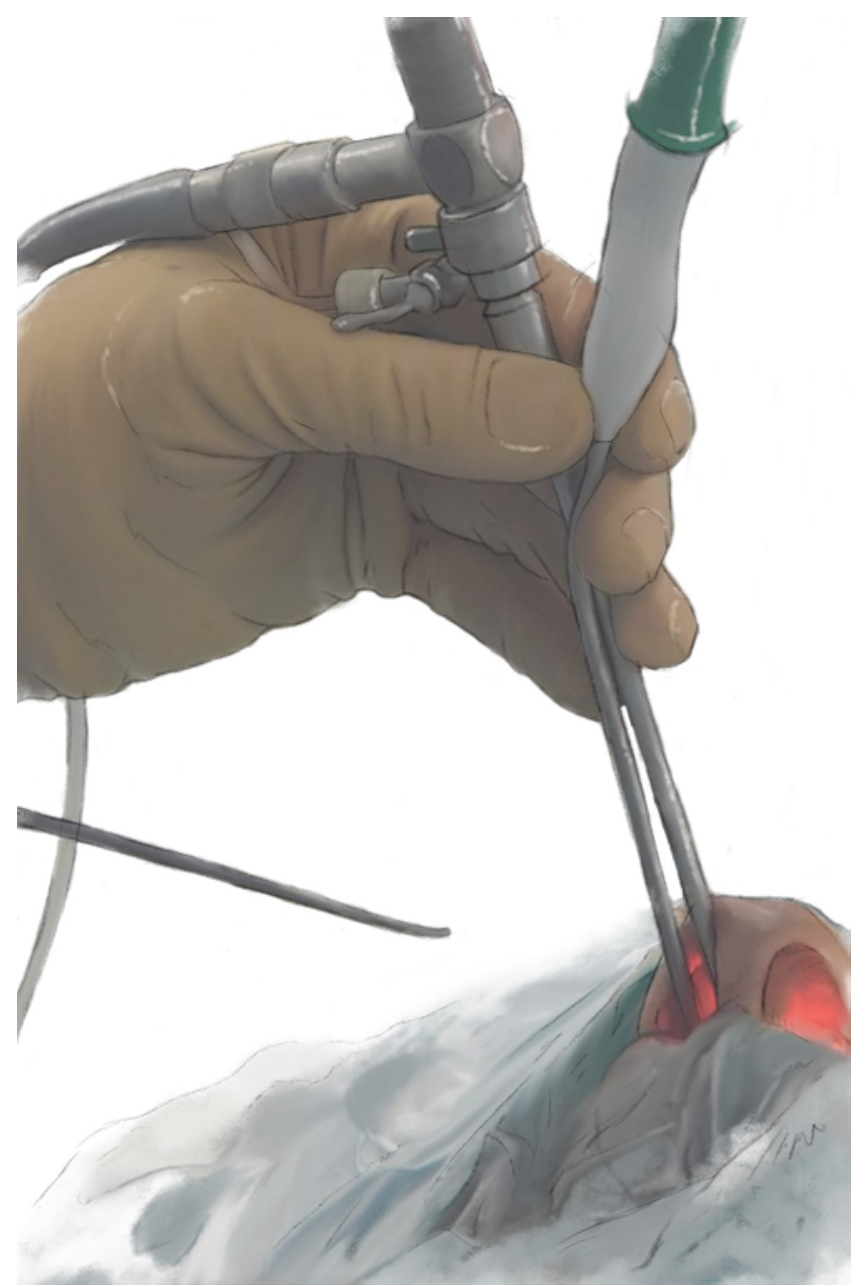

FIG. 1. Artistic depiction of the chopsticks technique, focusing on the finger grip required for holding both endoscope and suction with the nondominant hand. Copyright Carlo Serra. Published with permission.

(immediately postoperatively, at discharge, and 3 months postoperatively), and pre- and postoperative high-field 3 T MRI (preoperatively, 3 months postoperatively, and yearly thereafter). Biochemical remission was defined as normalization of hypersecretion into the normal reference range as defined by accepted international guidelines., ${ }^{4,11}$ Three-tesla intraoperative MRI (iMRI) is routinely performed unless medically contraindicated or logistically not possible as previously described.$^{30}$ The frequency of assessment beyond 3 months postoperatively depends on secretory status and endocrinological and surgical outcomes. Preoperative and postoperative rhinological assessment with an olfaction test (12-item Sniffin' Sticks [Burghart] odor identification test ${ }^{33}$ ) and the Sino-Nasal Outcome Test-20 (SNOT-20) ${ }^{25}$ is also part of the pre- and postoperative evaluation. Patient data are treated according to the ethical standards of the Declaration of Helsinki as approved by our institutional committee (Cantonal Ethics Committee Zurich).

To have a homogeneous cohort, only data of those patients who underwent transsphenoidal surgery (TSS) for a PA with both the chopsticks technique and 3T iMRI 
were included. Neurosurgical, endocrinological, neuroradiological, and rhinological follow-ups at 3 months were also required. The invasiveness of the adenoma was classified according to the modified Knosp classification, ${ }^{23}$ whereas an extension of adenoma in the suprasellar space was assessed according to the classification of Hardy. ${ }^{12}$ Adenomas deemed as not invasive according to the dichotomized Knosp classification (Knosp grade $\leq 2$ ) were initially considered for gross-total resection (GTR). The likelihood of resection was preoperatively assessed also using the Zurich Pituitary Score. ${ }^{32}$ The volume and diameter of adenomas were measured on pre-, intra-, and postoperative 3T MRI with the iPlan software (iPlan Cranial, Brainlab). The extent of resection (EOR) was calculated as the percentage-wise reduction of residual tumor volume to baseline tumor volume on preoperative MRI as previously described..$^{30}$

\section{Surgical Protocol and Technique}

All TSSs are performed under general anesthesia with orotracheal intubation. The patient is kept supine with the head fixed in an MR-compatible head-fixation system (Noras Head Holder, NORAS MRI Products $\mathrm{GmbH}$ ), and the operating table is placed in a $20^{\circ}$ reverse-Trendelenburg position. The operating surgeon faces the patient on the patient's right side, while the scrub nurse stands on the left side. The monitor and endoscopic equipment (2D endoscope Karl Storz GmbH \& Co.; since January 2017, Xion $\mathrm{GmbH}$ ) are positioned opposite the surgeon, next to the patient's head.

We use the chopsticks technique according to the same principles described in detail by Froelich's group. $.^{15} \mathrm{Sim}-$ ply put, the endoscope is held with the nondominant hand and is balanced between the proximal phalange of the first and second finger and the fourth finger (Fig. 1). The distal phalanges of the first, second, and third fingers of the same hand can therefore be used to hold and move the suction, with the nasal structures themselves partly supporting the instruments. The dominant hand is free and can be used to work with any needed surgical instrument (Fig. 2).

The surgical steps are standardized and performed as follows: we usually choose a right-sided transnasal, mononostril approach unless the nasal anatomy suggests or imposes a different approach. After having identified the choana and the overlying recessus sphenoethmoidalis, the natural sphenoid ostium is reached superiorly. The posterior part of the nasal septum is submucosally fractured to expose the contralateral sphenoid ostium. A sphenoidotomy is performed as needed with removal of the sphenoidal septa (if present) to obtain sufficient exposure of the anterior anatomy of the sella. The exposure reaches from the clival recess to the planum sphenoidalis in a craniocaudal direction and laterolaterally from one carotid bulging to the other. Both opticocarotid recesses are identified. The anterior wall of the sella is then opened, exposing inferiorly the sellar floor and reaching superiorly up to the limbus sphenoidalis. The medial margins of both cavernous sinuses are also included in the exposure. The dura mater is subsequently incised in a cruciform fashion, reaching the cavernous sinus laterally in order to inspect both medial walls of the cavernous sinuses. After the neu-

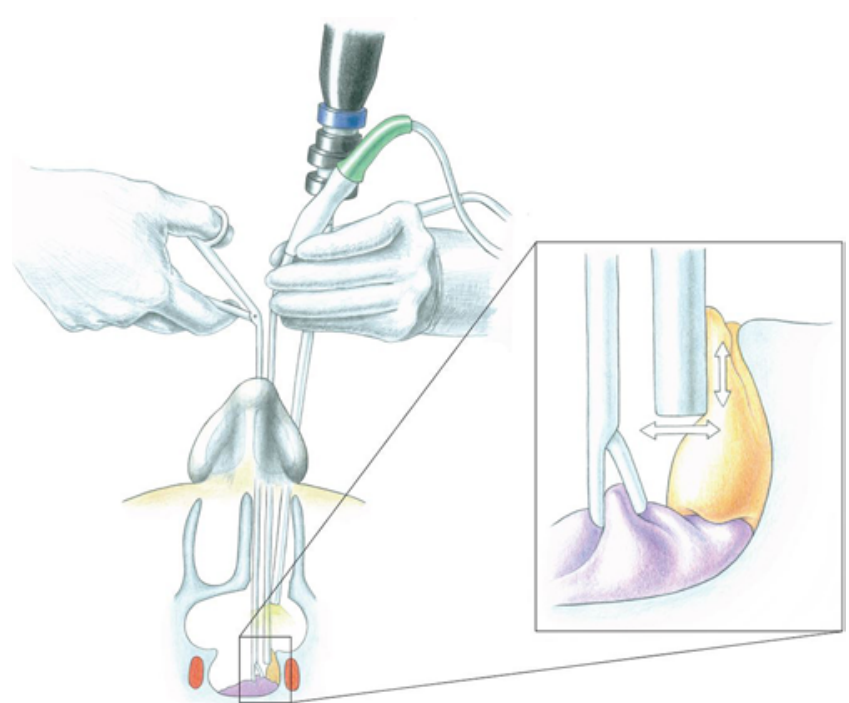

FIG. 2. Artistic depiction of the chopsticks technique, focusing on the surgical technique. The suction can be used not only to keep the surgical field clean but also as a working tool for dissection. The distal phalanges of the first 3 fingers can move the suction longitudinally and orthogonally to the optic with little effort since the nasal structures partly support the instruments. The dominant hand is then available to work with any needed surgical instrument. Copyright Carlo Serra. Published with permission.

rosurgeon decides that the resection is complete or feels that further inspection or resection is not safe enough, 3T iMRI is performed (3T Skyra VD13, Siemens) after going through the $3 \mathrm{~T}$ iMRI safety checklist. ${ }^{35}$ In case of residual tumor on 3T iMRI, endoscopic inspection and removal is performed if feasible. At the end of surgery, sellar reconstruction is performed depending on the presence of a CSF leak according to a 4-tier protocol. ${ }^{33}$

\section{Results}

From October 2012 to January 2020, 231 operations for a PA were performed at our institution. Of these, 156 operations (144 patients) fulfilled the inclusion criteria. Exclusion criteria are illustrated in Fig. 3, whereas characteristics of the included patients are described in detail in Table 1.

\section{Resection Results}

GTR and subtotal resection (STR) were the surgical goals in 122 and 34 operations, respectively. However, whenever deemed intraoperatively safely possible, GTR was attempted even in cases initially thought to be invasive.

In patients in whom GTR was the surgical target (tGTR), GTR was achieved in $106(86.9 \%)$ of 122 operations. GTR was achieved in $5(14.7 \%)$ of 34 operations in which STR was the initial goal (tSTR). On 3-month postoperative 3T MRI, the average EOR was $98.7 \%$ for the tGTR and $89.6 \%$ for the tSTR groups, respectively. In the entire cohort, GTR was achieved in 111 (71.2\%) of 156 operations with an average EOR of $96.7 \% 3$ months after surgery. The average residual adenoma volume was 0.29 


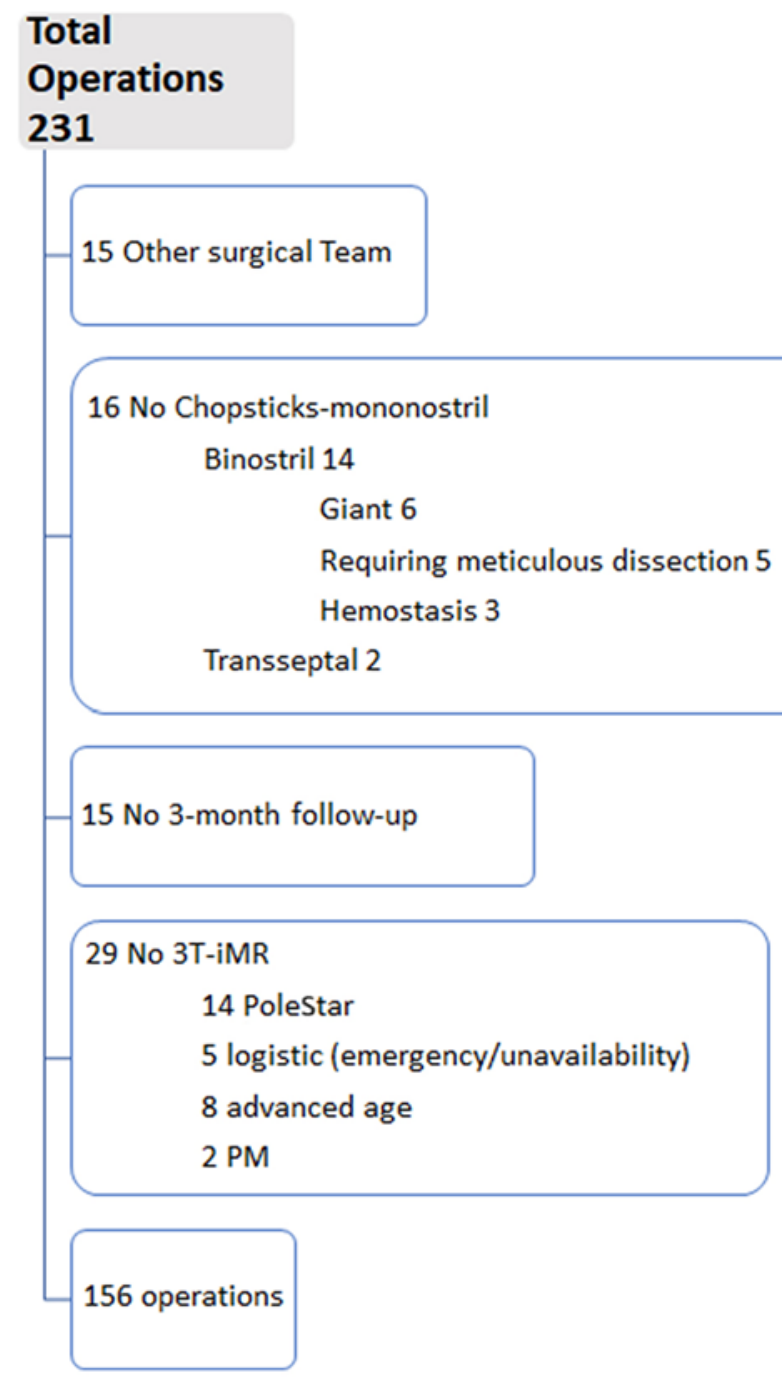

FIG. 3. Overview of patient series, excluded patients, and reason for exclusion. $\mathrm{PM}=$ pacemaker.

$\mathrm{cm}^{3}\left(0.06 \mathrm{~cm}^{3}\right.$ in the tGTR group and $1.11 \mathrm{~cm}^{3}$ in the tSTR group).

In the entire cohort, GTR was achieved in $42.0 \%$ of patients on 3T iMRI, and in $71.2 \%$ on 3-month postoperative MRI. In the tGTR group, GTR changed from 59 (48.4\%) of 122 operations on 3T iMRI to $106(86.9 \%)$ of 122 on 3-month postoperative MRI, whereas in the tSTR group, GTR changed from $3(8.8 \%)$ of 34 to $6(17.6 \%)$ of 34 on 3-month postoperative MRI.

\section{Clinical Outcome}

At the last clinical follow-up (median 15 months, range 3-70 months), $26.3 \%$ of patients had an improvement of at least 1 hormonal axis, whereas $11.5 \%$ of patients had a new pituitary single-axis deficit, the thyrotropin axis being the most likely to be affected. Complete endocrine remission was achieved in 79.6\% of patients (43/54 patients: growth hormone-producing adenoma in 28/36 patients; prolactinoma in 8/11 patients; adrenocorticotropic hormone-pro-
TABLE 1. Baseline patient and adenoma characteristics

\begin{tabular}{|c|c|}
\hline Variable & Value \\
\hline Sex, male/female & $83 / 73$ \\
\hline Median age, yrs (range) & $54(21-83)$ \\
\hline Median adenoma volume, $\mathrm{cm}^{3}$ (range) & $3.4(0.03-33.7)$ \\
\hline \multicolumn{2}{|l|}{ Knosp grade } \\
\hline 0 & 31 \\
\hline 1 & 41 \\
\hline 2 & 44 \\
\hline $3 \mathrm{~A}$ & 26 \\
\hline 3B & 6 \\
\hline 4 & 8 \\
\hline \multicolumn{2}{|l|}{ Zurich Pituitary Score } \\
\hline 1 & 39 \\
\hline II & 81 \\
\hline III & 29 \\
\hline IV & 7 \\
\hline \multicolumn{2}{|l|}{ Adenoma type } \\
\hline NFPA & 102 \\
\hline ACTH & 4 \\
\hline $\mathrm{GH}$ & 36 \\
\hline PRL & 11 \\
\hline TSH & 1 \\
\hline Pluri & 2 \\
\hline
\end{tabular}

$\mathrm{ACTH}=$ adrenocorticotropic hormone-producing adenoma; $\mathrm{GH}=$ growth hormone-producing adenoma; NFPA = nonfunctioning pituitary adenoma; pluri = plurihormonal adenoma; $\mathrm{PRL}$ = prolactinoma; $\mathrm{TSH}$ = thyrotropin-secreting adenoma.

Values are number of adenomas unless noted otherwise.

ducing in 4/4 patients; thyrotropin-secreting adenoma in $1 / 1$ patient; and plurihormonal adenoma in $2 / 2$ patients) with a secreting adenoma.

There was no mortality in the series. Only 1 patient had permanent postoperative neurological morbidity at the last follow-up (visual field defect). Postoperative CSF leaks occurred in $5(3.2 \%)$ cases, 3 of them requiring surgical repair (2 nasoseptal flaps). Medical complications occurred in 9 patients: 1 patient had a pulmonary embolism, 2 patients had CSF infection requiring antibiotic therapy, 1 patient developed an intracranial intraparenchymal abscess (requiring surgical evacuation), and a further patient had a sinusitis. Lastly, 4 patients had transient electrolyte imbalance (syndrome of inappropriate antidiuretic hormone secretion or diabetes insipidus) requiring medical treatment.

There were no patients with epistaxis. At a median rhinological follow-up of 18 months (range 3-58 months), new hyposmia (defined as a Sniffin' Sticks test $<9 / 12$ points) occurred in 3 (3.2\%) of 95 cases, whereas new anosmia (defined as a Sniffin' Sticks test $\leq 6 / 12$ points) occurred in 2 (2.1\%) of 95 cases. Evaluation of postoperative rhinological function with SNOT-20 revealed only 1 patient $(1.1 \%)$ with a severe postoperative impairment (SNOT-20 score $>40$ ).

Three patients had new postoperative hyposmia. One 
patient had severe impairment of sinonasal function (SNOT-20 score $>40$ ). The operation resulted in endocrine remission in $76.9 \%$ of patients with secreting adenomas.

\section{Discussion}

In this study, we aimed to assess the surgical outcome of the chopsticks technique in TSS for PA. This purely endoscopic technique allows one surgeon to use 3 instruments simultaneously (the endoscope, the suction, and a working instrument) in the narrow space of a single nostril. In this way, a satisfactory bimanual dissection and a flexible dynamic view are possible without the need of a second surgeon or mechanical arm (Fig. 2). Additional manipulation of healthy nasal tissues for the purpose of lodging and moving surgical instruments (turbinectomy/ ethmoidectomy or a binostril approach) is also not required.

\section{Technical Nuances in TSS}

To our knowledge, there is no class I evidence demonstrating the superiority of a bimanual dissection (i.e., 3- or 4-hand technique) over a 2-hand technique in which only 1 hand is available for dissection. Intuitively, bimanual dissection appears safer, particularly if complex intraarachnoidal dissection is required, or in cases of extensive bleeding. Typically, either a second surgeon holding the endoscope or a mechanical arm is needed to assist the first surgeon, so that he or she can use both hands to carry out the operation. In case of a 3- or 4-hand technique, a welltrained team effort is required to guarantee a successful and time-efficient workflow. Moreover, this implies the presence of either 2 surgeons, which might not always be feasible, or a mechanical arm to hold the endoscope. However, a mechanical arm cannot be adjusted as quickly or as flexibly to continuously offer the surgeon the most suitable view. The chopsticks technique, on the contrary, allows the preservation of a satisfactory bimanual dissection without the need to compromise the surgical view flexibility or the need for additional human resources.

Similarly, no data unequivocally demonstrate that a binostril approach would allow better surgical results than a pure mononostril approach. According to recent literature, binostril access would guarantee better maneuverability of instruments and a more panoramic view of the targeted surgical area.,9,10 This does not, however, appear to translate into an improved outcome, neither in terms of EOR nor in terms of morbidity. ${ }^{9}$ We do completely agree with authors who suggest that a binostril approach can be advantageous in the case of profuse bleeding or of a particularly large tumor with a significant perisellar extension, since it can offer a wider view. We also think that lesions requiring extensive and meticulous dissection are also not ideal candidates for the chopstick technique. In fact, in some highly selected cases in our series, we chose a binostril approach at the beginning of the surgery because of either the size or location of the adenoma (Fig. 3). Such extreme cases, however, are rare in pituitary surgery. However, if needed (as could happen in case of major vessel injury), surgery can be swiftly converted from a mononostril into a binostril approach. For example, in
5 patients harboring an adenoma within the cavernous sinus in close contact with the internal carotid artery, we chose to convert the procedure to a 3-hand binostril approach to improve and speed up the dissection. In more patients, we converted the procedure for a similar reason: to quickly control massive intraoperative venous bleeding. As a matter of fact, our resection results, in terms of both GTR and EOR, compare favorably with published results and support the efficacy of the chopsticks technique. One may speculate that this might be favored by the systematic use of 3T iMRI, which allows for excellent intraoperative assessment of the $\mathrm{EOR}^{30}$ and of intra- and perisellar anatomical structures. ${ }^{31,34}$ It is possible that the use of 3T iMRI compensates for the partially reduced view created by the mononostril approach. The intraoperative accurate volumetric assessment of the EOR and the precise view of intra- and perisellar anatomy provided by the 3T iMRI give the surgeon immediate feedback on the results of surgery. The surgeon can calibrate the surgical strategy according to the information visible on 3T iMRI and thus, if required, reformulate surgical goals, as well as decide if adjunctive risks are to be taken or if more additional surgical maneuvers are really needed. It derives that the invasiveness of the surgical approach is no more fixed a priori for every single case but adapted during the surgical procedure depending on the intraoperative information. As attractive as this hypothesis might be, it is very difficult to ascertain if the use of intraoperative imaging does really improve the GTR rate or complication rate. ${ }^{30}$ Randomized prospective studies are needed to assess if the use of 3T iMRI would be helpful in clarifying this point and to ascertain if the chopstick technique is truly superior compared with other mono- and binostril techniques.

\section{Surgical Morbidity}

Any surgical intervention should maximize preservation of healthy tissue, unless it is indisputably proven that the benefits of an increased invasiveness of a procedure outweigh its potential harm. For this reason, we chose a mononostril approach with the aim to manipulate the least amount of healthy nasal mucosa as possible. Some authors have suggested that the insertion of fewer tools per nasal fossa, as is the case in the binostril technique, would be associated with less surgical trauma to the nasal epithelium. ${ }^{7}$ On the other hand, a recent meta-analysis concerning this topic demonstrated a higher incidence of epistaxis in patients undergoing binostril approaches..$^{36}$

We reported new hyposmia in $1.9 \%$ of patients and only 1 case of a SNOT-20 score $>40$ points. Our results concerning surgical morbidity seem thus to be at least in line, if not even better, with the available literature. ${ }^{1,2,8,14,17,18,26}$ It must be underlined that all data of the present study are extracted from a prospective registry ${ }^{29}$ a fact that notoriously leads to more precise data acquisition and thus very often to reports of higher incidences of complications. According to a recent review on the topic,,$^{18}$ new moderate to severe hyposmia would occur in up to $39.7 \%$ of patients operated on with a microscopic approach and in 3\% of patients operated on with an endoscopic technique. These reported data, however, should be interpreted very cautiously, since they result from pooling results out of a highly 
selected number of papers with few patients included (149 pooled patients in the endoscopic cohort). Moreover, there is a marked heterogeneity of methods used to assess olfactory function among studies. Similar considerations apply also when reviewing the literature for sinonasal morbidity after TSS. Several different questionnaires and scores are adopted (Anterior Skull Base Questionnaire, ${ }^{21}$ Anterior Skull Base Nasal Inventory-12, ${ }^{16}$ SNOT-20, nasal obstruction symptom evaluation, and SF-36 ${ }^{26}$ ), thereby rendering comparisons difficult.

Lastly, the somehow more cumbersome bimanual dissection due to the chopsticks technique did not result in our series in an increased general morbidity. The incidence of endocrinological, neurological, and medical complications in our series also is comparable with the incidence commonly reported in the literature. ${ }^{1,2}$

\section{Conclusions}

The endoscopic mononostril, 2-hand, 3-instrument chopsticks technique allows a single surgeon to preserve satisfactory bimanual dissection within a single nostril, thereby reducing the surgical impact on the nasal cavities, and possibly resulting in reduced sinonasal morbidity. General morbidity did not exceed that commonly reported in the literature. GTR and EOR results in the present series compared favorably with most available literature, notwithstanding a somewhat more cumbersome bimanual dissection.

\section{Acknowledgments}

The authors would like to thank Mr. Nicola Podda for Fig. 1 and Ms. Lisa Cuthbertson for Fig. 2.

\section{References}

1. Agam MS, Wedemeyer MA, Wrobel B, Weiss MH, Carmichael JD, Zada G: Complications associated with microscopic and endoscopic transsphenoidal pituitary surgery: experience of 1153 consecutive cases treated at a single tertiary care pituitary center. J Neurosurg 130:1576-1583, 2019

2. Ammirati M, Wei L, Ciric I: Short-term outcome of endoscopic versus microscopic pituitary adenoma surgery: a systematic review and meta-analysis. J Neurol Neurosurg Psychiatry 84:843-849, 2013

3. Barker FG II, Klibanski A, Swearingen B: Transsphenoidal surgery for pituitary tumors in the United States, 1996-2000: mortality, morbidity, and the effects of hospital and surgeon volume. J Clin Endocrinol Metab 88:4709-4719, 2003

4. Biller BM, Grossman AB, Stewart PM, Melmed S, Bertagna $\mathrm{X}$, Bertherat J, et al: Treatment of adrenocorticotropin-dependent Cushing's syndrome: a consensus statement. J Clin Endocrinol Metab 93:2454-2462, 2008

5. Cappabianca P, Cavallo LM, Colao A, Del Basso De Caro M, Esposito F, Cirillo S, et al: Endoscopic endonasal transsphenoidal approach: outcome analysis of 100 consecutive procedures. Minim Invasive Neurosurg 45:193-200, 2002

6. Cappabianca P, Cavallo LM, de Divitiis E: Endoscopic endonasal transsphenoidal surgery. Neurosurgery 55:933-941, 2004

7. Castelnuovo P, Pistochini A, Locatelli D: Different surgical approaches to the sellar region: focusing on the "two nostrils four hands technique." Rhinology 44:2-7, 2006

8. Chaaban MR, Chaudhry AL, Riley KO, Woodworth BA: Objective assessment of olfaction after transsphenoidal pituitary surgery. Am J Rhinol Allergy 29:365-368, 2015
9. Conrad J, Ayyad A, Wüster C, Omran W, Weber MM, Konerding MA, et al: Binostril versus mononostril approaches in endoscopic transsphenoidal pituitary surgery: clinical evaluation and cadaver study. J Neurosurg 125:334-345, 2016

10. Elhadi AM, Hardesty DA, Zaidi HA, Kalani MY, Nakaji P, White WL, et al: Evaluation of surgical freedom for microscopic and endoscopic transsphenoidal approaches to the sella. Neurosurgery 11 (Suppl 2):69-79, 2015

11. Giustina A, Chanson P, Kleinberg D, Bronstein MD, Clemmons DR, Klibanski A, et al: Expert consensus document: a consensus on the medical treatment of acromegaly. Nat Rev Endocrinol 10:243-248, 2014

12. Hardy J, Wigser SM: Trans-sphenoidal surgery of pituitary fossa tumors with televised radiofluoroscopic control. J Neurosurg 23:612-619, 1965

13. Jho HD, Carrau RL, Ko Y, Daly MA: Endoscopic pituitary surgery: an early experience. Surg Neurol 47:213-223, 1997

14. Kim BY, Son HL, Kang SG, Kim SW, Hong YK, Jeun SS, et al: Postoperative nasal symptoms associated with an endoscopic endonasal transsphenoidal approach. Eur Arch Otorhinolaryngol 270:1355-1359, 2013

15. Labidi M, Watanabe K, Hanakita S, Park HH, Bouazza S, Bernat AL, et al: The chopsticks technique for endoscopic endonasal surgery-improving surgical efficiency and reducing the surgical footprint. World Neurosurg 117:208-220, 2018

16. Little AS, Kelly D, Milligan J, Griffiths C, Prevedello DM, Carrau RL, et al: Predictors of sinonasal quality of life and nasal morbidity after fully endoscopic transsphenoidal surgery. J Neurosurg 122:1458-1465, 2015

17. Little AS, Kelly DF, Milligan J, Griffiths C, Prevedello DM, Carrau RL, et al: Comparison of sinonasal quality of life and health status in patients undergoing microscopic and endoscopic transsphenoidal surgery for pituitary lesions: a prospective cohort study. J Neurosurg 123:799-807, 2015

18. Majovsky M, Astl J, Kovar D, Masopust V, Benes V, Netuka D: Olfactory function in patients after transsphenoidal surgery for pituitary adenomas-a short review. Neurosurg Rev 42:395-401, 2019

19. Manickavasagam J, Segaram S, Harkness P: Functional endoscopic sinus surgery chopstick technique. Laryngoscope 120:975-977, 2010

20. May M, Hoffmann DF, Sobol SM: Video endoscopic sinus surgery: a two-handed technique. Laryngoscope 100:430432, 1990

21. McCoul ED, Bedrosian JC, Akselrod O, Anand VK, Schwartz TH: Preservation of multidimensional quality of life after endoscopic pituitary adenoma resection. J Neurosurg 123:813-820, 2015

22. Messerklinger W: Endoscopy of the Nose. Urban \& Schwarzenberg, 1978

23. Micko AS, Wöhrer A, Wolfsberger S, Knosp E: Invasion of the cavernous sinus space in pituitary adenomas: endoscopic verification and its correlation with an MRI-based classification. J Neurosurg 122:803-811, 2015

24. Mortini P: Cons: endoscopic endonasal transsphenoidal pituitary surgery is not superior to microscopic transsphenoidal surgery for pituitary adenomas. Endocrine 47:415-420, 2014

25. Piccirillo JF, Merritt MG Jr, Richards ML: Psychometric and clinimetric validity of the 20-Item Sino-Nasal Outcome Test (SNOT-20). Otolaryngol Head Neck Surg 126:41-47, 2002

26. Pledger CL, Elzoghby MA, Oldfield EH, Payne SC, Jane JA Jr: Prospective comparison of sinonasal outcomes after microscopic sublabial or endoscopic endonasal transsphenoidal surgery for nonfunctioning pituitary adenomas. J Neurosurg 125:323-333, 2016

27. Pratheesh R, Rajaratnam S, Prabhu K, Mani SE, Chacko G, Chacko AG: The current role of transcranial surgery in the 
management of pituitary adenomas. Pituitary 16:419-434, 2013

28. Rolston JD, Han SJ, Aghi MK: Nationwide shift from microscopic to endoscopic transsphenoidal pituitary surgery. Pituitary 19:248-250, 2016

29. Sarnthein J, Stieglitz L, Clavien PA, Regli L: A patient registry to improve patient safety: recording general neurosurgery complications. PLoS One 11:e0163154, 2016

30. Serra C, Burkhardt JK, Esposito G, Bozinov O, Pangalu A, Valavanis A, et al: Pituitary surgery and volumetric assessment of extent of resection: a paradigm shift in the use of intraoperative magnetic resonance imaging. Neurosurg Focus 40(3):E17, 2016

31. Serra C, Maldaner N, Muscas G, Staartjes V, Pangalu A, Holzmann D, et al: The changing sella: internal carotid artery shift during transsphenoidal pituitary surgery. Pituitary 20:654-660, 2017

32. Serra C, Staartjes VE, Maldaner N, Muscas G, Akeret K, Holzmann D, et al: Predicting extent of resection in transsphenoidal surgery for pituitary adenoma. Acta Neurochir (Wien) 160:2255-2262, 2018

33. Soyka MB, Serra C, Regli L, Meier E, Holzmann D: Longterm olfactory outcome after nasoseptal flap reconstructions in midline skull base surgery. Am J Rhinol Allergy 31:334337,2017

34. Staartjes VE, Stricker S, Muscas G, Maldaner N, Holzmann $\mathrm{D}$, Burkhardt JK, et al: Intraoperative unfolding and postoperative pruning of the pituitary gland after transsphenoidal surgery for pituitary adenoma: a volumetric and endocrinological evaluation. Endocrine 63:231-239, 2019

35. Stienen MN, Fierstra J, Pangalu A, Regli L, Bozinov O: The Zurich checklist for safety in the intraoperative magnetic resonance imaging suite: technical note. Oper Neurosurg (Hagerstown) 16:756-765, 2019
36. Wen G, Tang C, Zhong C, Li X, Li J, Li L, et al: Mononostril versus binostril endoscopic transsphenoidal approach for pituitary adenomas: a systematic review and meta-analysis. PLoS One 11:e0153397, 2016

\section{Disclosures}

The authors report no conflict of interest concerning the materials or methods used in this study or the findings specified in this paper.

\section{Author Contributions}

Conception and design: Serra. Acquisition of data: Serra, Holzmann, Soyka, Gilone, Schmid, Tschopp, Regli. Analysis and interpretation of data: Serra. Drafting the article: Serra, Staartjes, Maldaner, Regli. Critically revising the article: Serra, Schmid, Tschopp, Regli. Reviewed submitted version of manuscript: Serra, Regli. Approved the final version of the manuscript on behalf of all authors: Serra. Statistical analysis: Serra. Administrative/ technical/material support: Serra. Study supervision: Serra, Regli.

\section{Supplemental Information \\ Videos}

Video 1. https://vimeo.com/402135603.

\section{Correspondence}

Carlo Serra: Clinical Neuroscience Center, University Hospital of Zürich, University of Zürich, Switzerland. carlo.serra@usz.ch. 